\title{
RR Lyrae star distance scale and kinematics from inner bulge to $50 \mathrm{kpc}$
}

\author{
Andrei Dambis ${ }^{1, \star}$, Leonid Berdnikov ${ }^{1,2, \star \star}$, Eva Grebel ${ }^{3, \star \star \star}$, Alexey Kniazev $^{1,4,5,6, \star \star \star \star}$, \\ Igor Katkov ${ }^{1, \dagger}$, and Tesfaye Dagne ${ }^{2, \ddagger}$ \\ ${ }^{1}$ Sternberg Astronomical Institute, M.V.Lomonosov Moscow State University, Universitetskii pr. 13, Moscow, \\ 119992 Russia \\ ${ }^{2}$ Astronomy and Astrophysics Research division, Entoto Observatory and Research Center, P.O. Box 8412, \\ Addis Ababa, Ethiopia \\ ${ }^{3}$ Astronomisches Rechen-Institut Zentrum für Astronomie der Universität Heidelberg, Germany \\ ${ }^{4}$ South African Astronomical Observatory, P.O. Box 9, Observatory, Cape Town, 7935, South Africa \\ ${ }^{5}$ Southern African Large Telescope, P.O. Box 9, Observatory, Cape Town, 7935, South Africa \\ ${ }^{6}$ Special Astrophysical Observatory of RAS, Nizhnij Arkhyz, Karachai-Circassia 369167, Russia
}

\begin{abstract}
We use the currently most complete sample of 3500 type ab RR Lyraes in our Galaxy with available radial-velocity and $[\mathrm{Fe} / \mathrm{H}]$ measurements to perform a statisticalparallax analysis for a subsample of $\sim 600$ type ab RR Lyraes located within $5 \mathrm{kpc}$ from the Sun to refine the parameters of optical and WISE W1-band period-metallicityluminosity relations and adjust our preliminary distances. The new zero point implies the rescaled estimates for the solar Galactocentric distance $\left(R_{G}=7.99 \pm 0.37 \mathrm{kpc}\right)$ and the LMC distance modulus $\left(D M_{\mathrm{LMC}}=18.39 \pm 0.09\right)$. We use the kinematic data for the entire sample to explore the dependence of the halo and thick-disk RR Lyrae velocity ellipsoids on Galactocentric distance from the inner bulge out to $R \sim 50 \mathrm{kpc}$.
\end{abstract}

\section{Introduction}

The high luminosities and old age make RR Lyrae type variables excellent distance indicators and kinematic tracers, in particular, for the halo and the thick disk subsystems of our Galaxy. Hence building a large enough sample of these variables having precise and homogeneous photometry, homogeneous metallicity estimates, and bona fide radial velocities is a task of prime importance. Until recently, only about 400 plus RR Lyraes in the extended solar neighborhood had all these data available $([3,4])$, and other spectroscopically measured Galactic variables of this type have been observed within the framework of SDSS survey and are mostly very distant objects. The situation with spectroscopic observations of RR Lyraes at intermediate distances has improved recently with the public

\footnotetext{
${ }^{\star}$ dambis@yandex.ru

$\star \star$ lberdnikov@yandex.ru

$\star \star \star$ grebel@ari.uni-heidelberg.de

$\star \star \star \star$ akniazev@saao.ac.za

$\dagger$ katkov.ivan@gmail.com

†tesfayedagne7@gmail.com
} 
releases of LAMOST survey. To expand the spectroscopically observed RR Lyrae sample in the extended solar neighborhood we launched a dedicated program with the purpose of acquiring spectra for the greatest possible number of RR Lyraes to complement our long-term program of photometric observations of these variables. Here we briefly describe the data obtained and report some results concerning the RR Lyrae distance scale and halo kinematics based on our measurements combined with published data including those provided by huge SDSS and LAMOST spectroscopic surveys.

\section{The sample and data}

Our sample consists of all 3444 type ab RR Lyrae variables for which we could find published radialvelocity measurements or measure radial velocities from the spectra we acquired with the Southern African Large Telescope (SALT) in 2015-2016.

\subsection{Radial velocities and metallicities}

The adopted RR Lyrae radial velocities and metallicities come from a number of sources. First, our own spectroscopic data with 320 measurements for 300 stars including 260 stars with no prior spectroscopic data available. Our observations cover spectral range of 3900-5000 , using grating GR1300 and slit with 1.25 arcsec width. It gives us a possibility to detect Balmer lines $(\mathrm{H} \delta, \mathrm{H} \gamma$, $\mathrm{H} \beta$ ) and Ca II K (3924 ̊) line to use S-method for metallicity determination and produces spectral resolution $R=1300-1500$. All data are very standard long-slit and are reduced automatically with our pipeline based on MIDAS and IRAF programs. Second, the published results of dedicated spectroscopic studies for about 400 stars compiled in our paper ([4]), third, measurements available in public releases of SDSS ([1]), LAMOST ([15]), and BRAVA ([9]) surveys (about 2800 stars). Typical errors of systemic $V_{R}$ values inferred from these data range from few to $60 \mathrm{~km} / \mathrm{s}$ (we show their distribution in Fig. 1) with a mean value of $\sim 22 \mathrm{~km} / \mathrm{s}$. Figure 2 shows the distribution of $[\mathrm{Fe} / \mathrm{H}]$ values for stars of our sample (transformed to the Zinn \& West [16] scale).

\subsection{Photometry and photometric distances}

We estimated the provisional distances of most of the stars of our sample based on the W1-band midinfrared light curves acquired as a result of spaceborne WISE all-sky photometric survey ([13]) and the corresponding period-luminosity relation of Dambis et al. ([5]):

$$
\left\langle M_{W 1}\right\rangle=-0.814-2.381 \cdot \log P_{F}+0.106 \cdot[\mathrm{Fe} / \mathrm{H}]_{\mathrm{ZW}} .
$$

We determined the distances to stars in heavily obscured bulge regions based on OGLE VI photometry ([10]) and the $V$-band metallicity-luminosity relation of Dambis et al. ([4]) consistent with the above W1-band PLZ relation:

$$
\left\langle M_{V}\right\rangle=+1.094+0.232 \cdot[\mathrm{Fe} / \mathrm{H}]_{\mathrm{ZW}} .
$$

Finally, we determined the distances to faint stars outside the bulge region with no or unreliable WISE data (discovered mostly as a result of an analysis of Catalina Sky Survey data $[6,11]$ ) from their Gaia G-band photometry using the following PLZ relation derived in the same way as the corresponding $W 1$-band relation that we reported in [5]:

$$
\left\langle M_{G}\right\rangle=-0.550-1.531 \cdot \log P_{F}+0.140 \cdot[\mathrm{Fe} / \mathrm{H}]_{\mathrm{ZW}} .
$$

Interstellar extinction was taken into account either using the extinction map ([7]) or appropriate period-metallicity-color relations. 


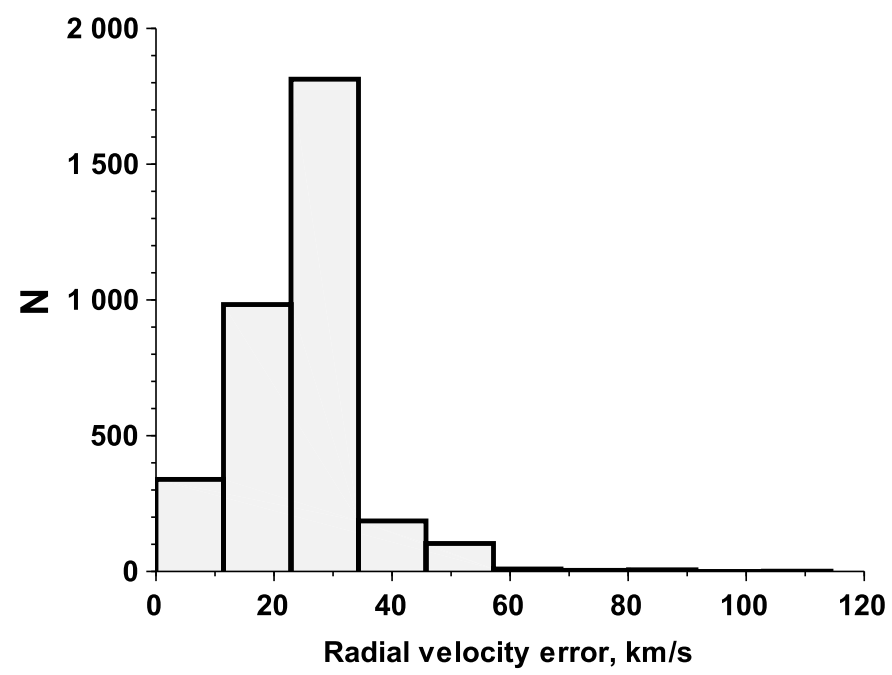

Figure 1. Distribution of errors of systemic $V_{R}$ values for type ab RR Lyrae variables of our sample.

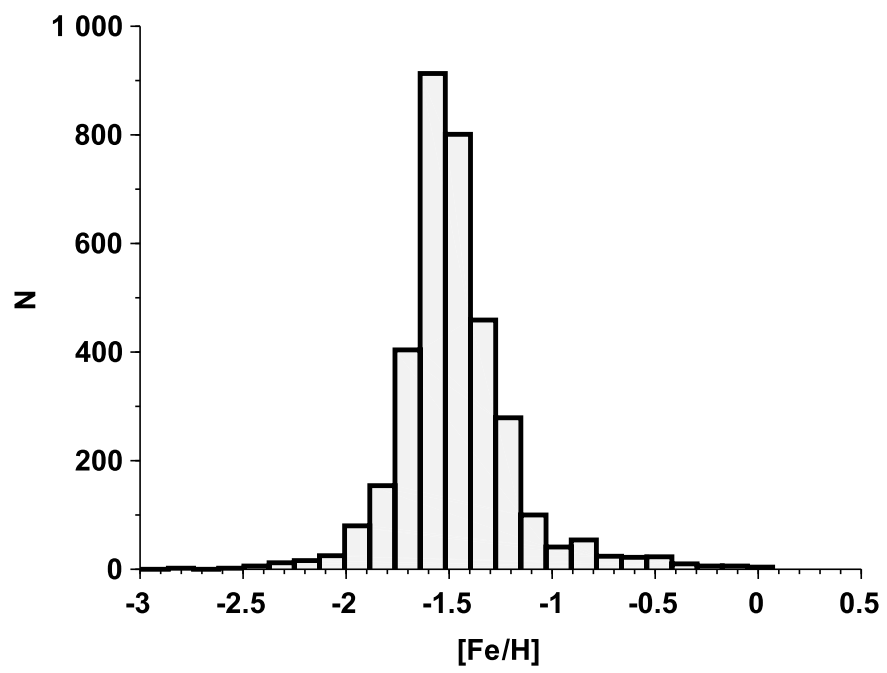

Figure 2. Distribution of $[\mathrm{Fe} / \mathrm{H}]$ values for type ab RR Lyrae variables of our sample transformed to the Zinn \& West scale. 
Table 1. Kinematical parameters of RR Lyrae populations in the extended solar neighborhood and the refined WISE $W 1$-band PLZ relation.

\begin{tabular}{lrrrrrrr}
\hline Population & Sample fraction & $U_{0}$ & $V_{0}$ & $W_{0}$ & $\sigma V_{R}$ & $\sigma V_{\varphi}$ & $\sigma V_{\theta}$ \\
\cline { 3 - 8 } & $(\alpha$ and 1- $\alpha)$ & \multicolumn{7}{c}{$\mathrm{km} / \mathrm{s}$} \\
\hline Halo & 0.766 & -1.8 & -214.6 & -6.0 & 163.6 & 94.7 & 88.4 \\
& \pm 0.028 & \pm 8.0 & \pm 7.5 & \pm 4.4 & \pm 6.9 & \pm 4.7 & \pm 3.8 \\
\hline Disc & 0.234 & -14.4 & -36.5 & -18.6 & 46.5 & 37.8 & 27.9 \\
& \pm 0.028 & \pm 5.5 & \pm 5.4 & \pm 3.6 & \pm 5.5 & \pm 4.9 & \pm 3.4 \\
\hline$M_{W 1}=-0.885+2.381 \cdot \log P+0.106 \cdot[\mathrm{Fe} / \mathrm{H}]$ & \pm 0.060 \\
\hline
\end{tabular}

\subsection{Proper motions}

Our sources of proper motions are the APOP ([12], used for most of the stars at Galactic latitudes $\left.|b|>27^{\circ}\right)$, HSOY ([2]), and UCAC4 ([14]) catalogs.

\section{Results}

\subsection{Distance scale and local kinematics}

We first used the subsample of 610 stars located within $5 \mathrm{kpc}$ from the Sun drawn from our initial sample to refine the zero point of our WISE W1-band PLZ relation ([5]) and estimate the kinematical parameters (mean velocities and parameters of velocity dispersion ellipsoid) of the halo and thick-disk RR Lyrae populations in the solar neighborhood using the bimodal method of statistical parallax (see $[3,4]$ for details). The results are summarized in Table 1.

Our refined WISE $W 1$-band PLZ zero point is brighter than the value derived in [4, 5] implying the rescaled estimates for the solar Galactocentric distance $\left(R_{G}=7.99 \pm 0.37 \mathrm{kpc}\right)$ and the LMC distance modulus $\left(D M_{\mathrm{LMC}}=18.39 \pm 0.09\right)$, which agree well with most of the current estimates.

\subsection{Velocity ellipsoid of the halo}

We then fix the mean velocity components of halo RR Lyrae stars at the values listed in the first row of Table 1 and assume the halo velocity ellipsoid to be two-axial (i.e., we set $\sigma V_{\varphi}=\sigma V_{\theta}$ ) to explore the variation of the radial component of halo velocity ellipsoid $\left(\sigma V_{R}\right)$ with Galactocentric distance $R_{G}$. We show the resulting dependence in Figure 3. The main feature of the radial velocity dispersion behavior is that it decreases steadily from $\sim 180 \mathrm{~km} / \mathrm{s}$ to $\sim 100 \mathrm{~km} / \mathrm{s}$ in the Galactocentric distance interval $R_{G}=3-16 \mathrm{kpc}$ and remains practically constant at greater distances at least out to $R_{G} \sim 50 \mathrm{kpc}$, which mostly agrees with the results of Kafle et al. ([8]) based on blue horizontal-branch star data.

Acknowledgments: This work makes use of observations from the South African Astronomical Observatory(SAAO) and Southern African Large Telescope (SALT), supported by the National Research Foundation of South Africa, and data from the SDSS and LAMOST projects. The data reduction of all data was supported by the Russian Science Foundation (project no. 14-50-00043), and the light-curve analysis was supported by the Russian Science Foundation (project no. 14-22-00041). 


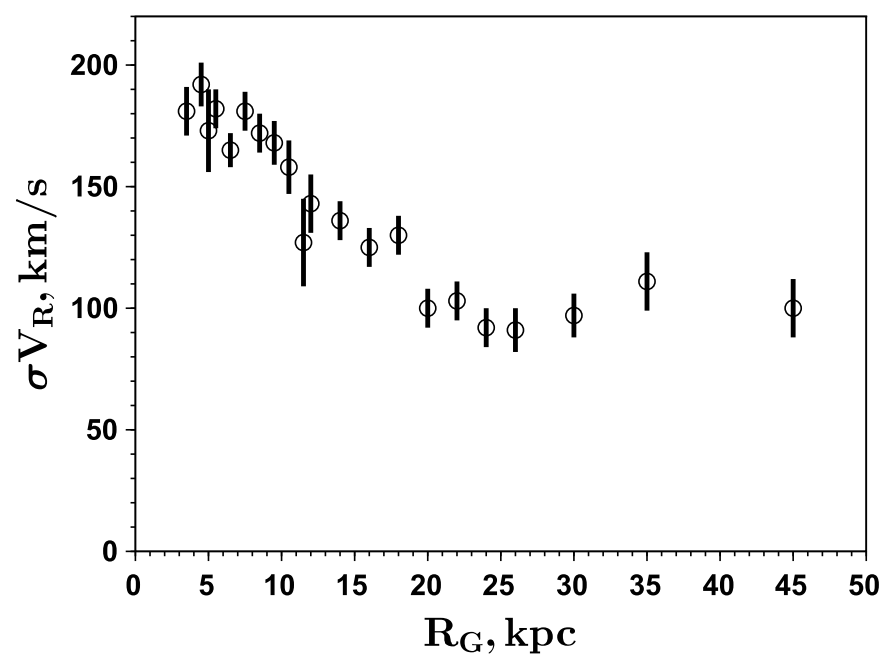

Figure 3. Radial velocity dispersion for halo RR Lyraes as a function of Galactocentric distance.

\section{References}

[1] Albareti, F. D., Allende Prieto, C., Almeida, A., et al., preprint (arXiv: 1608.02013) (2016)

[2] Altmann, M., Roeser, S., Demleitner, M., Bastian, U., \& Schilbach, E., A\&A, 600, L4 (2017)

[3] Dambis, A. K., MNRAS, 396, 553 (2009)

[4] Dambis, A. K., Berdnikov, L. N., Kniazev, A. Yu., Kravtsov, V. V., Rastorguev, A. S., Sefako, R., \& Vozyakova, O. V., MNRAS, 435, 3206 (2013)

[5] Dambis, A. K., Rastorguev, A. S., \& Zabolotskikh, M. V., MNRAS, 439, 3765 (2014)

[6] Drake, A. J., Catelan, M., Djorgovski, S. G., et al., ApJ, 763, 32 (2013)

[7] Drimmel, R., Cabrera-Lavers, A., López-Corredoira, M., A\&A, 409, 205 (2003)

[8] Kafle, P. R., Sharma, S., Lewis, G. F., \& Bland-Hawthorn, J., ApJ, 761, 98 (2012)

[9] Kunder, A., Rich, R. M., Koch, A., et al., ApJL, 821, L25 (2016)

[10] Soszyński, I., Udalski, A., Szymański, M. K., et al., AcA, 64, 177 (2014)

[11] Torrealba, G., Catelan, M., Drake, A. J., et al., MNRAS, 446, 2251 (2015)

[12] Qi, Z., Yu, Y., \& Bucciarelli, B., AJ, 150, 137 (2015)

[13] Wright, E. L., Eisenhardt, P. R. M., Mainzer, A. K., et al., AJ, 140, 1868 (2010)

[14] Zacharias, N., Finch, C. T., Girard, T. M., et al., AJ, 145, 44 (2013)

[15] Zhao, G., Zhao, Y.-H., Chu, Y.-Q., Jing, Y.-P., \& Deng, L.-C., RA\&A, 12, 723 (2012)

[16] Zinn, R., \& West, M. J., ApJS, 55, 45 (1984) 